\title{
Dual Vortex Theory of Strongly Interacting Electrons: A Non-Fermi Liquid with a Twist
}

\author{
Leon Balents \\ Room 1D-368, Bell Labs, Lucent Technologies, 700 Mountain Ave, Murray Hill, NJ 07974 \\ Matthew P. A. Fisher \\ Institute for Theoretical Physics, University of California, Santa Barbara, CA 93106-4030 \\ Chetan Nayak \\ Physics Department, University of California, Los Angeles, CA 90095-1547
}

(September 17, 2018)

\begin{abstract}
As discovered in the quantum Hall effect, a very effective way for strongly-repulsive electrons to minimize their potential energy is to aquire non-zero relative angular momentum. We pursue this mechanism for interacting two-dimensional electrons in zero magnetic field, by employing a representation of the electrons as composite bosons interacting with a Chern-Simons gauge field. This enables us to construct a dual description in which the fundamental constituents are vortices in the auxiliary boson fields. The resulting formalism embraces a cornucopia of possible phases. Remarkably, superconductivity is a generic feature, while the Fermi liquid is not - prompting us to conjecture that such a state may not be possible when the interactions are sufficiently strong. Many aspects of our earlier discussions of the nodal liquid and spin-charge separation find surprising incarnations in this new framework.
\end{abstract}

PACS numbers: 74.20.Mn, 71.10.Hf, 71.27.+a, 74.72.-h

\section{INTRODUCTION}

Fermi liquid theory is the cornerstone of the modern theory of metals, as well as band theories of insulators and semiconductors. This theory - like most perturbative theories - is informed by the assumption that the kinetic energy is the dominant scale. As a result, the theory is constructed in momentum space, where the kinetic energy is diagonalized. This leads to strong kinematic constraints which circumscribe corrections to the underlying free fermion behavior. In this paper, our point of departure is a different extreme limit in which the interaction must be dealt with at the outset, and the kinematic constraints might, consequently, be inoperative. Hence, we are forced to adopt a non-perturbative approach. As is often the case in non-perturbative problems - for example the quantum Hall effect and the one-dimensional electron gas - it is advantageous to adopt a real space approach. Here, this also enables us to gain a vantage point from which to focus on the strong electron interaction.

The past few decades have witnessed the discovery of a number of physical systems in which the interaction energy is comparable to or greater than the kinetic energy. These materials exhibit strange behavior which is not readily captured within the conventional Fermi liquid framework. The high- $T_{c}$ cuprate superconductors 3 are the most famous example, but there are certainly others, such as heavy=fermion materials 1 and high-mobility 2DEGs at large $r_{s}$. 0 . Ironically, even ${ }^{3} \mathrm{He}-$ the birthplace of Fermi liquid theory - falls in this category 6 . The analysis of such systems may require an approach of the type propounded in this paper.

Following the above reasoning, we are led to search for a means of incorporating strong electron-electron repulsion from the outset. As discovered by Laughlin, the spatial separation due to the centrifugal barrier for nonzero angular momentum is a very effective way for particles to lower their Coulomb energy. We consider strongly interacting electrons moving in the two-dimensional (2d) continuum, and assume that these strong interactions include a hard-core which prevents the crossing of electron trajectories. Some of the resulting physics is reminiscent of the quantum Hall effect: pairs of particles tend to spin around one another. There are, however, some significant differences: time-reversal symmetry is not explicitly broken, and further, the kinetic energy is not quenched. Nevertheless, our injestigations in the remainder of this paper and elsewherel lead us to suspect that strongly repulsive electrons in the $2 \mathrm{~d}$ continuum can form a p-wave $\left(p_{x} \pm i p_{y}\right)$ superconductor!

To develop a low-energy effective field theory, we first use only the non-crossing constraint on the fermion world-lines. This is a sufficient condition to allow the use of statistical transmutation 6 to realize up and downspin electrons as bosonic fields interacting with a ChernSimons gauge field which attaches flux to spin. Without additional assumptions, we can then pass to a dual theory of vortices in the up- and down-spin bosonic fields. In this way, we argue that many of our previous results on the nodal liquid 1 . 23 hold with a much wider range of validity. With this approach, we believe that we gain an unfettered view of the entire phase diagram of this infinitely strongly interacting fermionic system.

Since our dual theory is of Ginzburg-Landau form, its 
phase structure can be analyzed by considering the condensation of various fields. If no vortex field condenses, the system is superconducting with a non-zero angular momentum pairing state with $p_{x}+i p_{y}$ symmetry 13 . Since vortex condensation typically implies charge ordering, it is usually driven by a periodic potential or long-range Coulomb interactions. In their absence we thus conjecture that the generic state of the stronglyinteracting system is superconducting. This is an astonishing conclusion, given the lack of a palpable 'pairing mechanism'. Evidently, strongly repulsive interactions essentially force electrons of opposite spin to "rotate" about one another and introduce strong superconducting correlations. Other ordered phases result when the vortex fields condense. For example, a spin- or chargedensity wave results if both the up- and down-spin vortex fields condense.

A basic feature of any superconductor is spin-charge separation 14. To access spin-charge separation in the $p_{x}+i p_{y}$ superconductor within our dual GinzburgLandau formulation requires consideration of "paired" vortex composites. When these composite bosons condense, they can destroy the superconductivity - but spincharge separation survives. Specifically, if a vortex in the down-spin boson field pairs with a vortex in the up-spin boson field and this pair condenses, translational symmetry is spontaneously broken by the formation of a crystalline state of spinless charge $e$ solitons. The spin sector is gapped except for chiral edge states, so that this phase is a $T$-violating nodal liquid (i.e. a chiral spin liquid). Alternatively, if a vortex in the down-spin boson field pairs with an anti-vortex in the up-spin boson field and this pair condenses, a transition occurs into a fully-gapped superconductor, such as a superconductor with tightlybound pairs. If both types of vortex pairs condense, an analogous spin-liquid results. In each of these phases, time reversal invariance is spontaneously broken.

With the inclusion of an ionic potential acting on the electrons, however, $T$-invariant phases are possible, and expected. Indeed, by allowing for terms in the dual Ginzburg-Landau theory which break rotational invariance, gapped modes in the spin sector can go soft at finite momentum. For a uniaxial potential, gapless modes naturally appear at two points in momentum space. At these two points the vortex-anti-vortex field is critical, and can be conveniently re-fermionized as two Dirac fields. These can be identified as the nodal quasiparticles of a $p_{x}$ superconducting phase. Similarly, an ionic potential with square symmetry leads to four low-energy points in momentum space, and thereby a fourfold Dirac theory recovering the spectrum of $d_{x^{2}-y^{2}}$ quasiparticles (see below and Sec. VI for a discussion of some subtleties of the d-wave case). Within our theory, a (very) strong local repulsion acting in concert with an ionic potential with square symmetry are the essential ingredients for high temperature $d$-wave superconductivity. In the $a b$ sence of the ionic potential, strong $T$-violating pairing with $p_{x}+i p_{y}$ symmetry is expected. This is the pairing symmetry in the A-phase of a superfluid 3-He film 15.

An appealing feature of our dual Ginzburg-Landau formulation is that it gives a clear meaning to spincharge separation (and spin-charge confinement) in twodimensional electron systems. Indeed, we identify an Ising-like $Z_{2}$ symmetry which when unbroken leaves spin and charge separate. Spin-charge confinement is driven by an Ising ordering transition.

Remarkably, although our theory is intimately tied to a real-space picture, Fermi surface physics is not lost, as evidenced by the nodal quasiparticles. The Fermi liquid phase itself is much more elusive! It occurs, if at all, only as a narrow region analogous to a (fermionic) "entangled flux liquid" in the Ginzburg-Landau theory, and is certainly only possible if the $Z_{2}$ symmetry is broken. However, it is unlikely that such a state can be obtained in the Ginzburg-Landau theory. It is here that we speculate the relative angular momentum between electrons plays an important role. Indeed, (very singular) s-wave scattering states are known to dominate the physics of the Fermi liquid with hard-core radius $a \ll 1 / k_{F}$. We suspect that the inclusion of such singular wavefunctions invalidates the passage to a local and analytic vortex lagrangian. See Sec. VI for further discussion of this point.

The phase diagram which results from this analysis contains a plethora of fascinating states, including superconducting states of pairing symmetry $p_{x}, p_{x}+i p_{y}$, $d_{x^{2}-y^{2}}, d_{x y}$, and their quantum disordered counterparts. These states are characterized by a separation between the characteristic scales of the charge and spin and even - when the $Z_{2}$ symmetry is unbroken - true spin-charge separation. A particularly salient aspect of this phase diagram is the miniscule domain, and we speculate perhaps even absence, of the Fermi lifyid state. This seems to support the claims of Anderson 16 and co-workers that Fermi liquid theory breaks down in strongly-interacting two-dimensional systems (though it does not support their bold claims that Fermi liquid theory breaks down even at weak-coupling). The proofs of the stability of Fermi liquid theory at weak-coupling 17 presumably do not apply to the strongly-interacting limit which we have considered.

In section II, we first discuss the statistical transmutation which obviates the need for a local pair field. Constructing a dual theory, we describe the standard antiferromagnetic and charge-density-wave states which result from the condensation of single vortices. In section III, we discuss paired vortices and the $Z_{2}$ symmetry which distinguishes their condensation from that of individual vortices. When this symmetry is unbroken, spin and charge separate. In section IV, we discuss the phase diagram which results from the condensation of paired vortices. This phase diagram revolves about a $p_{x} \pm i p_{y}$ superconducting state. In section $\mathrm{V}$, we show how timereversal $(\mathrm{T})$ invariant superconducting states such as $p_{x}$ or $d_{x y}$ can arise in this model. The momentum-space structure and concomitant phenomenology of BCS-like $d_{x y}$ or $d_{x^{2}-y^{2}}$ superconductors is recovered. We find 
that the physics of the nodal liquid reappears in a new guise: the nodons are vortices in a vortex field whose fermionic statistics result from their interaction with a Chern-Simons gauge field. In chapter VI, we arrive, ultimately, at a phase diagram which is the synthesis of ideas of duality and vortex condensation common to field theories of the quantum Hall effect as well as our earlier work on nodal liquids, but is almost entirely orthogonal to the underlying conceit of Fermi liquid theory.

\section{FERMIONS, FLUX ATTACHMENT, AND DUALITY}

We focus throughout on spinful electrons moving in the two-dimensional continuum, interacting via a spinindependnet interaction. We assume that the electronelectron repulsion is strong enough that no two electrons can ever be coincident. Precisely this "hard-core" constraint makes it possible to transform the interacting twodimensional electron gas into a mathematically equivalent system of interacting spinful bosons, by attaching "statistical" flux with an appropriate Chern-Simons gauge field. Such a "bosonization" scheme for $2 \mathrm{~d}$ spinless electrons has been particularly illuminatipo in the context of the fractional quantum Hall effect 10 . With spin there is considerable freedom in how one attaches the flux tubes to convert fermions into bosons. We adopt a scheme in which flux is attached to the spin of the electrons, and define

$$
c_{\alpha}(\boldsymbol{r})=b_{\alpha}(\boldsymbol{r}) \exp \left[i e_{\alpha} \int_{\boldsymbol{r}^{\prime}} \Theta\left(\boldsymbol{r}-\boldsymbol{r}^{\prime}\right) 2 S^{z}\left(\boldsymbol{r}^{\prime}\right)\right],
$$

with a "charge" $e_{\uparrow}=1$ and $e_{\downarrow}=-1$. Here

$$
S^{z}(\boldsymbol{r})=\left[n_{\uparrow}(\boldsymbol{r})-n_{\downarrow}(\boldsymbol{r})\right] / 2
$$

is the z-component of the spin density operator with $n_{\alpha}=c_{\alpha}^{\dagger} c_{\alpha}=b_{\alpha}^{\dagger} b_{\alpha}$ (no sum on $\alpha$ ), and $\Theta(\boldsymbol{r})$ denotes the angle that $\boldsymbol{r}$ makes with the $x$-axis. The boson operators satisfy canonical commutators, $\left[b_{\alpha}(\boldsymbol{r}), b_{\beta}^{\dagger}\left(\boldsymbol{r}^{\prime}\right)\right]=0$ for $\boldsymbol{r} \neq \boldsymbol{r}^{\prime}$. Due to the non-crossing constraint, the "onsite" commutators need not be specified.

An advantage of the above scheme for flux attachment is that with zero total spin, $S_{\text {tot }}^{z}=0$ (as assumed hereafter), the statistical flux "seen" by the Chern-Simons bosons vanishes on average. For spinless electrons this happy situation requires the presence of a strong external magnetic field (as in the FQHE, c.f. Ref. 17 As for Abelian bosonization in one spatial dimensiont 0 , the above choice of a spin quantization axis masks the underlying spin-rotational invariance. But as we shall see, it is possible to restore explicit $\mathrm{SU}(2)$ symmetry by a subsequent "refermionization".

After transforming to boson operators, the partition function for the Hamiltonian of $2 \mathrm{~d}$ interacting electrons can be expressed as a functional integral over bosonic fields and a statistical gauge field, $\alpha_{\mu}$, with associated (Euclidean) Lagrangian density:

$$
\mathcal{L}=b_{\alpha}^{*}\left(\partial_{\tau}-i e_{\alpha} \alpha^{0}\right) b_{\alpha}-\frac{1}{2 m_{e}} b_{\alpha}^{*}\left(\nabla-i e_{\alpha} \boldsymbol{\alpha}\right)^{2} b_{\alpha}-\mathcal{L}_{c s} .
$$

In the "Coulomb gauge" $\partial_{i} \alpha^{i}=0$ the Chern-Simons term is $\mathcal{L}_{c s}=(i / 2 \pi) \alpha^{0}\left(\epsilon_{i j} \partial_{i} \alpha_{j}\right)$, but can be cast into a more convenient gauge invariant form:

$$
\mathcal{L}_{c s}\left(\alpha_{\mu}\right)=i \frac{1}{4 \pi} \epsilon_{\mu \nu \lambda} \alpha_{\mu} \partial_{\nu} \alpha_{\lambda}
$$

The form of the electron interaction term (not shown) is unchanged under "bosonization" due to the equivalence of the fermion and boson densities: $c^{\dagger} c=b^{\dagger} b$.

We now implement the standard 2+1-dimensional duality transformation 18 which exchanges bosons (the $b_{\alpha}$ 's) for vortices in the bosonic fields - arriving at a description in terms of vortex field operators, denoted $\Phi_{\alpha}$. To illuminate this, it is instructive to briefly consider an alternate representation in terms of boson world lines:

$$
\mathcal{L}_{w l}=\frac{1}{2}\left(J_{\mu}^{\alpha}\right)^{2}+i e_{\alpha} J_{\mu}^{\alpha} \alpha_{\mu}-\mathcal{L}_{c s}\left(\alpha_{\mu}\right) .
$$

Here $J_{\mu}^{\alpha}$ denotes a bosonic three-current (with $\mu$ running over $2+1$ space-time coordinates) for spin component $\alpha$. The first term measures the length of the space-time world-lines and represents the kinetic energy. The ChernSimons coupling generates a sign change when two world lines exchange, transforming to fermions. To implement duality, these three-currents are expressed in terms of two gauge fields, $a_{\mu}^{\alpha}$, one for each spin component:

$$
J_{\mu}^{\alpha}=\frac{1}{2 \pi} \epsilon_{\mu \nu \lambda} \partial_{\nu} a_{\lambda}^{\alpha} .
$$

In this way, charge conservation $\left(\partial_{\mu} J_{\mu}^{\alpha}=0\right)$ is automatically satisfied. The dual Lagrangian can be obtained by inserting this expression into $\mathcal{L}_{w l}$, and integrating out the Chern-Simons field, $\alpha^{\mu}$. Electron charge quantization is implemented by the vortex operators, $\Phi_{\alpha}$, which are minimally coupled to $a_{\mu}^{\alpha}$.

The final dual form consists of two Ginzburg-Landau theories, coupled via a Chern-Simons term:

$$
\begin{gathered}
\mathcal{L}_{\text {dual }}=\sum_{\alpha} \mathcal{L}_{G L}\left(\Phi_{\alpha}, a_{\mu}^{\alpha}\right)+\mathcal{L}_{c s}\left(a_{\mu}^{\uparrow}-a_{\mu}^{\downarrow}\right), \\
\mathcal{L}_{G L}\left(\Phi, a_{\mu}\right)=\frac{1}{2}\left|\left(\partial_{\mu}-i a_{\mu}\right) \Phi\right|^{2}+V(\Phi)+\frac{1}{2}\left(f_{\mu \nu}\right)^{2} .
\end{gathered}
$$

with a "potential" that can be expanded as $V(\Phi)=$ $r|\Phi|^{2}+u|\Phi|^{4}+\ldots$.

Since the Chern-Simons term only involves spin currents, it is extremely convenient to introduce charge and spin gauge fields: 


$$
a_{\mu}^{\rho}=a_{\mu}^{\uparrow}+a_{\mu}^{\downarrow} ; \quad a_{\mu}^{\sigma}=a_{\mu}^{\uparrow}-a_{\mu}^{\downarrow},
$$

(and corresponding field strengths, $f_{\mu \nu}^{\rho}, f_{\mu \nu}^{\sigma}$ ). As with Abelian bosonization in one dimension, charge and spin currents defined by $J_{\mu}^{\rho}=J_{\mu}^{\uparrow}+J_{\mu}^{\downarrow}$ and $J_{\mu}^{\rho}=J_{\mu}^{\uparrow}-J_{\mu}^{\downarrow}$, are given by derivatives of the charge and spin fields, respectively:

$$
J_{\mu}^{\rho}=\frac{1}{2 \pi} \epsilon_{\mu \nu \lambda} \partial_{\nu} a_{\lambda}^{\rho} ; \quad J_{\mu}^{\sigma}=\frac{1}{2 \pi} \epsilon_{\mu \nu \lambda} \partial_{\nu} a_{\lambda}^{\sigma} .
$$

Longer range Coulomb interactions can be readily incorporated by adding a term bilinear in the electron charge density: $\epsilon_{i j} \partial_{i} a_{j}^{\rho}$. In this dual representation, $\Phi_{\alpha}^{\dagger}$ creates a vortex in the electron wavefunction - effectively increasing the angular momentum of all spin $\alpha$ electrons by one unit. An important feature of $\mathcal{L}_{\text {dual }}$ is that there are precisely as many positive as negative circulation (electron) vortices (for each spin species), implying a relativistic form for $\Phi_{\alpha}$.

It is instructive to briefly mention how these dual fields couple to an external electromagnetic field, $A_{\mu}$. As usual $A_{\mu}$ couples directly to the total electrical current, $J_{\mu}^{\rho}$, so that from Eq. 10 one has:

$$
\mathcal{L}_{A}=\frac{1}{2 \pi} A_{\mu} \epsilon_{\mu \nu \lambda} \partial_{\nu} a_{\lambda}^{\rho}
$$

It is also convenient to introduce an external "spin" gauge field, $A_{\mu}^{\sigma}$, which couples to the total (z-component) spin current, $J_{\mu}^{\sigma}$ :

$$
\mathcal{L}_{A}^{\sigma}=\frac{1}{2 \pi} A_{\mu}^{\sigma} \epsilon_{\mu \nu \lambda} \partial_{\nu} a_{\lambda}^{\sigma} .
$$

The dual Ginzburg-Landau representation can be fruitfully employed to describe various possible phases of spinful $2 \mathrm{~d}$ electrons satisfying the 'non-crossing' constraint. For instance, imagine a phase in which the (electron) vortices are absent in the ground state (except as virtual fluctuations), which corresponds to taking $r$ large and positive in the above Ginzburg-Landau description. Being massive, $\Phi_{\alpha}$ can be safely integrated out, leaving an effective theory:

$$
\mathcal{L}_{e f f}=\frac{1}{2}\left(f_{\mu \nu}^{\rho}\right)^{2}+\mathcal{L}_{c s}\left(a_{\mu}^{\sigma}\right),
$$

which describes massless charge fluctuation, and spin fluctuations gapped (in the bulk) by the Chern-Simons term. This is a superconducting phase, which can be verified by noting that the pair field operator $\left(c_{\uparrow} c_{\downarrow}\right)$ creates a (2+1 space-time) monopole of strength two in the field $B_{\mu}^{\rho}=\epsilon_{\mu \nu \lambda} \partial_{\nu} a_{\lambda}^{\rho}$. Since the gauge field $a_{\mu}^{\rho}$ is massless, the energy cost to make a free monopole is finite (monopoles interact via $2+1$ Coulomb force), so that the pair-field exhibits true ODLRO. Due to the Chern-Simons term, this superconducting phase exhibits a quantized Hall "spin-conductance", $\sigma_{x y}^{s}=1$, a signature of a spontaneous breakdown of time reversal invariance. This follows by noting that the Lagrangian with spin "gauge" field, $\mathcal{L}_{\text {eff }}+\mathcal{L}_{A}^{\sigma}$, depends quadratically on both $a_{\mu}^{\rho}$ and $a_{\mu}^{\sigma}$, so that they can be integrated out to give,

$$
\mathcal{L}_{\text {eff }}+\mathcal{L}_{A}^{\sigma}=-i \sigma_{x y}^{s} \frac{1}{4 \pi} \epsilon_{\mu \nu \lambda} A_{\mu}^{\sigma} \partial_{\nu} A_{\lambda}^{\sigma},
$$

with $\sigma_{x y}^{s}=1$. Following the analysis in Ref. 19, one can readily verify that a $2 \mathrm{~d}$ BCS superconductor with $p_{x}+i p_{y}$ pairing symmetry has precisely such a value for the quantized spin conductance (also see below in Section IV). The spin state of the pair is then presumably a triplet with $s_{z}=0$. This is the phase of a $2 \mathrm{~d}$ superfluid 3-He A film.

Before discussing spin-charge separation, which is a generic property of a $2 \mathrm{~d}$ superconductor, it is instructive to consider phases described in the dual theory when vortices created by $\Phi_{\alpha}$ proliferate, and condense: $\left\langle\Phi_{\alpha}\right\rangle \neq 0$. As we shall see, in contrast to the $p_{x}+i p_{y}$ superconducting phase, these phases typically exhibit crystalline order, spontaneously breaking translational symmetry. To see this, note that upon vortex condensation, the dual "flux" $\epsilon_{i j} \partial_{i} a_{j}^{\alpha}$ is quantized in units of $2 \pi$, which corresponds to quantization of charge in units of the electron charge $e$. By analogy with the Abrikosov flux lattice phase of a Type II superconductor, one expects a breakdown of translational symmetry with spin up (and down) electrons forming an ordered lattice. Depending on the relative phase between the density wave of spin up and down electrons, this will be either a charge density wave $(\mathrm{CDW})$ state or an antiferromagnet $(\mathrm{AF})$. In the presence of a commensurate background periodic potential from the ions in the solid, one expects these density wave states to lock, resulting in insulating behavior.

These "crystalline" phases can presumably be energetically stabilized by a longer-range repulsive interaction between the electrons, in addition to the 'non-crossing' constraint (which is required to make our $2 \mathrm{~d}$ bosonization scheme legitimate). In their absence, our dual GinzburgLandau representation, (fortified by subsequent analysis below and physical reasoning) strongly suggests that the predominant ground state is the $p_{x}+i p_{y}\left(\right.$ or $p_{x}-i p_{y}$ ) superconductor. Given that spin-up and spin-down electrons prefer a state of non-vanishing relative angular momentum to minimize Coulomb repulsion, an $\ell= \pm 1$ orbital angular momentum state is clearly favored by the kinetic energy. As such, it seems that incorporating local Coulomb repulsion by forcing electrons of opposite spin into a relative angular momentum state is a very effective electronic mechanism for high temperature superconductivity!

\section{SPIN-CHARGE SEPARATION}

The phases described above are not the only possible phases for $2 \mathrm{~d}$ electrons satisfying the 'non-crossing' constraint. Composite order parameters can also condense, thereby leading to charge- and/or spin-insulators. We focus on the combinations 


$$
\Phi_{\rho}=\Phi_{\uparrow} \Phi_{\downarrow} ; \quad \Phi_{\sigma}=\Phi_{\uparrow} \Phi_{\downarrow}^{\dagger},
$$

which, as we shall see, are exceedingly interesting from a phenomenological standpoint. These order parameters can condense without breaking the $Z_{2}$ symmetry

$$
\Phi_{\uparrow, \downarrow} \rightarrow-\Phi_{\uparrow, \downarrow},
$$

so we can have $\left\langle\Phi_{\rho, \sigma}\right\rangle \neq 0$ while $\Phi_{\uparrow, \downarrow}=0$.

In the following we presume that $\Phi_{\rho}$ and $\Phi_{\sigma}$ describe the soft modes at low energies, and that $\Phi_{\alpha}$ remains massive. As we shall see, this leads naturally to a separation of low energy spin and charge degrees of freedom. Our motivation for this is two-fold. Firstly, spin-charge separation is a generic property of a superconductor such as the $p_{x}+i p_{y}$ state discussed above, and it is instructive to exhibit this separation within the present GinzburgLandau framework. But secondly, in many Mott insulators of interest the charge degrees of freedom freeze out at much higher energy scales than the energies on which local moments and spin order develops. This is typified by the undoped cuprate materials, with insulating behavior setting in on the scale of electron volts (the "Hubbard" $U)$ much higher than the antiferromagnetic ordering temperature. In order to capture these two very different energy scales within the present framework, it is essential to transform to the charge and spin vortex fields, $\Phi_{\rho}$ and $\Phi_{\sigma}$. Indeed, in the description of the antiferromagnetic insulator discussed above driven by condensation of $\Phi_{\alpha}$, charge ordering and local moment formation necessarily take place on the same energy scale, since the dual flux tubes in these vortex fields are electrons carrying both charge and spin.

Under the assumption that both fields $\Phi_{\alpha}$ remain massive, one can write down an effective theory for the soft modes $\Phi_{\rho, \sigma}$ by integrating out $\Phi_{\uparrow, \downarrow}$. Below we illustrate how this can be done, by regularizing the theory on a lattice. But more generally, the form of the effective theory is essentially dictated by symmetries, involving three contributions:

$$
\mathcal{L}_{\text {eff }}=\mathcal{L}_{\rho}+\mathcal{L}_{\sigma}+\mathcal{L}_{\text {int }},
$$

with a charge sector,

$$
\begin{gathered}
\mathcal{L}_{\rho}=\frac{1}{2}\left|\left(\partial_{\mu}-i a_{\mu}^{\rho}\right) \Phi_{\rho}\right|^{2}+r_{\rho}\left|\Phi_{\rho}\right|^{2}+u_{\rho}\left|\Phi_{\rho}\right|^{4} \\
+\frac{1}{2}\left(f_{\mu \nu}^{\rho}\right)^{2}+\frac{1}{2 \pi} A_{\mu} \epsilon_{\mu \nu \lambda} \partial_{\nu} a_{\lambda}^{\rho}
\end{gathered}
$$

a spin sector,

$$
\begin{aligned}
\mathcal{L}_{\sigma}== & \frac{1}{2}\left|\left(\partial_{\mu}-i a_{\mu}^{\sigma}\right) \Phi_{\sigma}\right|^{2}+r_{\sigma}\left|\Phi_{\sigma}\right|^{2}+u_{\sigma}\left|\Phi_{\sigma}\right|^{4} \\
& +\frac{1}{2}\left(f_{\mu \nu}^{\sigma}\right)^{2}+i \frac{1}{4 \pi} \epsilon_{\mu \nu \lambda} a_{\mu}^{\sigma} \partial_{\nu} a_{\lambda}^{\sigma}
\end{aligned}
$$

and sub-dominant interaction terms involving many derivatives (see eg. below). The charge sector has the Ginzburg-Landau form, with minimal coupling to the charge gauge field, $a_{\mu}^{\rho}$, and $A_{\mu}$ is the physical electromagnetic potential. The Chern-Simons term lives solely in the spin sector.

Some insight into the genesis of such a Lagrangian may be obtained by considering a lattice version of (8) and dropping the $a_{\mu}^{\uparrow, \downarrow}$ for simplicity. Writing $\Phi_{\alpha}=e^{\imath \theta_{\alpha}}$, we have

$$
S=\sum_{\langle i, j\rangle} \cos \left(\theta_{i}^{\alpha}-\theta_{j}^{\alpha}\right)
$$

where $i$ and $j$ denote sites of a $2 \mathrm{~d}$ (say square) lattice and a sum over $\alpha$ is understood. We now introduce charge and spin fields $\theta^{\rho, \sigma}$ :

$$
\theta^{\uparrow, \downarrow}=\frac{1}{2}\left(\theta^{\rho} \pm \theta^{\sigma}\right)+\frac{\pi}{2} s
$$

where $s= \pm 1$ is an Ising "spin" variable. By introducing $s$, we can treat $\theta^{\rho, \sigma}$ as angular variables since the action is invariant under $\theta^{\rho, \sigma} \rightarrow \theta^{\rho, \sigma}+2 \pi, s \rightarrow-s$. The action can then be rewritten as:

$$
S=\sum_{\langle i, j\rangle} s_{i} s_{j} \cos \frac{1}{2}\left(\theta_{i}^{\rho}-\theta_{i}^{\rho}\right) \cos \frac{1}{2}\left(\theta_{i}^{\sigma}-\theta_{i}^{\sigma}\right),
$$

since $\sin \frac{\pi}{2}\left(s_{i}-s_{j}\right)=0$ and $\cos \frac{\pi}{2}\left(s_{i}-s_{j}\right)=s_{i} s_{j}$. Let us now consider the effect of integrating out the $s_{i}$ 's. If we are in the symmetric phase in which the $Z_{2}$ is unbroken, this can be done perturbatively, as in the hightemperature expansion for the Ising model.

$$
\begin{aligned}
Z & =\sum_{s_{i}= \pm 1} e^{\sum_{i j} \beta J s_{i} s_{j}} \\
& =\sum_{S_{i}= \pm 1} \sum_{n} \frac{1}{n !}\left(\sum_{i j} \beta J s_{i} s_{j}\right)^{n}
\end{aligned}
$$

To leading order in $\beta$, which corresponds to decoupled free spins, one has $\left\langle s_{i} s_{j}\right\rangle=\delta_{i j}$, which implies an effective action of the form:

$$
\begin{aligned}
S_{\text {eff }}= & \sum_{\langle i, j\rangle}\left(1+\cos \left(\theta_{i}^{\rho}-\theta_{j}^{\rho}\right)\right)\left(1+\cos \left(\theta_{i}^{\sigma}-\theta_{i}^{\sigma}\right)\right) \\
= & \sum_{\langle i, j\rangle} \cos \left(\theta_{i}^{\rho}-\theta_{j}^{\rho}\right)+\cos \left(\theta_{i}^{\sigma}-\theta_{j}^{\sigma}\right) \\
& +\cos \left(\theta_{i}^{\rho}-\theta_{j}^{\rho}\right) \cos \left(\theta_{i}^{\sigma}-\theta_{j}^{\sigma}\right) .
\end{aligned}
$$

Upon making the identifications $\Phi_{\rho}=e^{i \theta_{\rho}}, \Phi_{\sigma}=e^{i \theta_{\sigma}}$ and restoring the gauge fields (minimally coupled) the first two terms are seen to be lattice versions of the continuum Ginzburg-Landau theories in $\mathcal{L}_{\rho}$ and $\mathcal{L}_{\sigma}$, respectively. The last term generates a gradient interaction term between the charge and spin sectors.

When the $Z_{2}$ symmetry is unbroken, as it is by assumption in (17), spin and charge separate, as we now argue. In the $p_{x}+i p_{y}$ superconducting state, which can 
be described by either (8) with $r_{\uparrow, \downarrow}>0$ or (17) with $r_{\rho, \sigma}>0$, the low-energy excitations are the gapless superfluid mode, $a_{\mu}^{\rho}$, which carries charge but no spin. At finite energy, there are also the quanta of $\Phi_{\sigma}$, which are fermionic and carry spin- $1 / 2$ by virtue of their coupling to the Chern-Simons gauge field, $a_{\mu}^{\sigma}$. They do not couple directly to the electromagnetic field, so we assign them quantum numbers $q=0, s=1 / 2$. As we shall see in the next section, these neutral fermionic spin $1 / 2$ excitations are the p-wave analog of "nodons", introduced in reference 11 for a d-wave superconductor.

When $r_{\rho}<0$ and $\Phi_{\rho}$ condenses, the dual flux $\epsilon_{i j} \partial_{i} a_{j}^{\rho}$ becomes quantized into "flux-tubes", by direct analogy with a Type II Ginzburg-Landau superconductor. Each one of these dual "flux tubes" carries one unit of electric charge, but no spin. We refer to these spinless charge $e$ solitons as "holons" 21. We thus see that provided the $Z_{2}$ symmetry in Eq. 16 is unbroken, spin and charge are separated. On the other hand, when the $Z_{2}$ symmetry is broken by the condensation of $\Phi_{\uparrow, \downarrow}$, the spin and charge are confined. Since $\Phi_{\uparrow, \downarrow}$ couples to $a_{\mu}^{\rho} \pm a_{\mu}^{\sigma}$, this condensation locks the spin and charge together, leaving only the electron in the spectrum.

To summarize, states of higher symmetry have less restricted spectra. The original dual representation in Eq. 8 has a $U(1) \times U(1)$ gauge symmetry, corresponding to independent rotations of $\Phi_{\uparrow}$ and $\Phi_{\downarrow}$;

$$
\Phi_{\alpha} \rightarrow \Phi_{\alpha} e^{i \Lambda_{\alpha}} ; \quad a_{\mu}^{\alpha} \rightarrow a_{\mu}^{\alpha}+\partial_{\mu} \Lambda_{\alpha},
$$

with two arbitrary functions $\Lambda_{\alpha}$. This gauge symmetry emerges when the conserved electron three-currents are expressed as a curl of the gauge fields, $a_{\mu}^{\alpha}$. Breaking down this large symmetry corresponds to "localization" or "quantization" of charge and/or spin. When the full symmetry is completely broken, both charge and spin become quantized together, and all the excitations have quantum numbers of the electron, with $\frac{q}{2}+s$ an integer (as in the antiferromagnetic insulator mentioned in Section II). But if this symmetry is only partially broken by condensation of $\Phi_{\rho}$ and $\Phi_{\sigma}$, leaving an unbroken $Z_{2}$, both charge $(e)$ and spin $(1 / 2)$ become quantized, but excitations exist with any combination of these quantum numbers. This will be nicely illustrated in the next Section where we employ the dual description, Eq. 17, to examine the properties of some T-breaking spin-charge separated phases.

\section{PHASES WITH BROKEN T}

Having established the form of Eq. 17 under the assumption of an unbroken $Z_{2}$ symmetry, and the concomitant spin-charge separation, we explore possible phases which emerge from this effective theory. We first focus on phases which (spontaneously) break time reversal invariance. As we shall see, these emerge naturally for electrons in jellium, moving in the absence of ionic potentials. In the following sections we consider the effects of ionic potentials which break rotational invariance and naturally drive transitions into time reversal invariant phases.

As already discussed, the phase in the absence of either spin or charge vortices is a $p_{x}+i p_{y}$ superconductor. Since the charge and spin sectors have effectively decoupled under the assumption of the unbroken $Z_{2}$ symmetry, it is possible to consider them separately. If the vortices in the charge sector proliferate and condense, $\left\langle\Phi_{\rho}\right\rangle \neq 0$, the field $\epsilon_{i j} \partial_{i} a_{j}^{\rho}$ becomes quantized in "dual" flux tubes, as discussed above. Each of these "flux" tubes carries charge $e$, but no spin. These charge $e$ spinless "holons" are expected to crystallize, by direct analogy with the Abrikosov flux-lattice. This charge $e$ crystal will presumably lock to any underlying ionic potential. With gapless spin-carrying edge states still present, this electrically insulating phase is the $p_{x}+i p_{y}$ analog of the nodal liquid. Once again, energetic stabilization of this crystalline phase presumably requires the presence of appreciable Coulomb repulsion between electrons on the scale of the mean electron spacing (in addition to the "noncrossing' constraint).

But suppose the spin vortices condense, in the absence of charge vortices? Due to the dual Anderson-Higgs mechanism, $a_{\mu}^{\sigma}$ becomes massive rendering the ChernSimons term ineffective, and leading to a spin-gap both in the bulk and at the edge. This implies "spin-insulating" behavior with $\sigma_{x y}^{s}=0$. How can we understand this fully spin-gapped superconducting phase? To this end it is convenient to briefly consider a BCS description of the quasiparticles in a $p_{x}+i p_{y}$ superconductor:

$$
\mathcal{H}_{B C S}=\sum_{\boldsymbol{k}} \epsilon_{\boldsymbol{k}} c_{\boldsymbol{k} \alpha}^{\dagger} c_{\boldsymbol{k} \alpha}+\Delta_{\boldsymbol{k}} c_{\boldsymbol{k} \uparrow}^{\dagger} c_{-\boldsymbol{k} \downarrow}^{\dagger}+\text { h.c. }
$$

with dispersion $\epsilon_{\boldsymbol{k}}=\left(k^{2} / 2 m_{e}\right)-\mu$ and gap function $\Delta_{\boldsymbol{k}}=v_{\Delta}\left(k_{x}+i k_{y}\right)$. In terms of a two-component spinor, $\psi_{1}(\boldsymbol{r})=c_{\uparrow}(\boldsymbol{r})$ and $\psi_{2}(\boldsymbol{r})=c_{\downarrow}^{\dagger}(\boldsymbol{r})$, this can be rewritten in the form of a Dirac equation with (Euclidean) Lagrangian:

$$
\mathcal{L}_{B C S}=\psi^{\dagger}\left[\partial_{\tau}+\tau^{z}\left(\left(-\partial_{j}^{2} / 2 m_{e}\right)-\mu\right)+i v_{\Delta} \tau^{j} \partial_{j}\right] \psi,
$$

with $j=x, y$. This gives the usual BCS quasiparticle dispersion: $E_{\boldsymbol{k}}= \pm \sqrt{\epsilon_{\boldsymbol{k}}^{2}+\left(v_{\Delta} k\right)^{2}}$. As in Ref. 11, one can define a gauge invariant charge neutral quasiparticle (a "nodon"), by transforming $\psi \rightarrow \exp \left(i \tau^{z} \varphi / 2\right) \psi$, with $\varphi$ the phase of the complex pair-field. Spin and charge are thereby separated, with the z-component of spin being the conserved U(1) "charge" in the Dirac theory: $2 S^{z}=$ $\psi^{\dagger} \psi$. Since the source field $A_{\mu}^{\sigma}$ couples to the conserved spin current, it can be readily incorporated into the above Dirac equation via a "minimal coupling" prescription: $\partial_{\mu} \rightarrow \partial_{\mu}-i A_{\mu}^{\sigma}$.

In the presence of a boundary, say at $y=0$ with boundary conditions $c_{\alpha}(x, y=0)=0$, one can readily 
show from the above Dirac theory that a chiral fermion edge mode exists only for positive chemical potential, $\mu>0$. In this BCS limit one clearly has $\sigma_{x y}^{s}=1$. But at very strong coupling when $\mu$ changessign, the ground state changes to a paired "molecular" 22 limit with zero $\sigma_{x y}^{s}=0$. Right at the transition, there are gapless bulk quasiparticle excitations described by a massless Dirac theory (at $\mu=0$ in Eq. 27) with a "node" at zero momentum. To access the molecular limit of the $p_{x}+i p_{y}$ superconductor presumably requires a very strong (and unphysical) attractive interaction between electrons, enabling up and down spin electrons to form a finite angular momentum bound state (with $\ell=1$ ). The attractive interaction must overcome the centrifugal repulsion between the two electrons (present due to the 'non-crossing' constraint).

A direct connection between the molecular $p_{x}+i p_{y}$ superconductor and the phase described by the dual theory when the spin vortex condenses, $\left\langle\Phi_{\sigma}\right\rangle \neq 0$, can be established by re-fermionizing the spin sector of the GinzburgLandau theory and showing its equivalence to the Dirac theory Eq. 27. To illustrate this we instead bosonize the Dirac theory. In the BCS limit of the $p_{x}+i p_{y}$ superconductor with $\mu$ positive, the massive relativistic Dirac fermion, $\psi$, can be converted to a relativistic boson, $\Phi$, via a Chern-Simons transformation:

$$
\begin{aligned}
\mathcal{L}_{B C S}= & \frac{1}{2}\left|\left(\partial_{\mu}-i a_{\mu}-i A_{\mu}^{\sigma}\right) \Phi\right|^{2}+M^{2}|\Phi|^{2}+U|\Phi|^{4} \\
& +\mathcal{L}_{c s}\left(a_{\mu}\right)-\mathcal{L}_{c s}\left(A_{\mu}^{\sigma}\right) .
\end{aligned}
$$

Here $M>0$ can be equated with the Dirac mass $\mu$. Indeed, the spectrum of this massive boson field, $\Phi$, is $\omega_{k}= \pm \sqrt{M^{2}+k^{2}}$ - the same form as the BCS quasiparticle dispersion, $E_{\boldsymbol{k}}$. Since $\Phi$ is massive it can be safely integrated out. The only remaining dependence on the source field $A_{\mu}^{\sigma}$ is through the last Chern-Simons term, which has been included to give the correct result for the spin Hall conductivity: $\sigma_{x y}^{s}=1$.

Remarkably, Eq. 28 precisely coincides with the spin sector of the dual Ginzburg-Landau theory, $\mathcal{L}_{\sigma}$ in Eq. 19. Indeed, with inclusion of the source term, $\mathcal{L}_{A}^{\sigma}$ in Eq. 12 , the full Lagrangian in the spin sector, $\mathcal{L}\left(A_{\mu}^{\sigma}\right)=\mathcal{L}_{\sigma}+\mathcal{L}_{A}^{\sigma}$ can be conveniently rewritten by shifting $a_{\mu}^{\sigma} \rightarrow a_{\mu}^{\sigma}+A_{\mu}^{\sigma}$ as,

$$
\begin{aligned}
\mathcal{L}_{\sigma}\left(A_{\mu}^{\sigma}\right)= & \frac{1}{2}\left|\left(\partial_{\mu}-i a_{\mu}^{\sigma}-i A_{\mu}^{\sigma}\right) \Phi_{\sigma}\right|^{2}+r_{\sigma}\left|\Phi_{\sigma}\right|^{2}+u_{\sigma}\left|\Phi_{\sigma}\right|^{4} \\
& +\mathcal{L}_{c s}\left(a_{\mu}\right)-\mathcal{L}_{c s}\left(A_{\mu}^{\sigma}\right)
\end{aligned}
$$

This is identical to $\mathcal{L}_{B C S}$ under the identification: $\Phi=$ $\Phi_{\sigma}, a_{\mu}=a_{\mu}^{\sigma}$ and $M^{2}=\mu^{2}=r_{\sigma}$. The upshot is that a simple re-fermionization of the spin sector, $\mathcal{L}_{\sigma}$, gives directly the BCS quasiparticle Lagrangian $\mathcal{L}_{B C S}$ in Eq. 26. The spin carrying but charge neutral vortex field, $\Phi_{\sigma}$, is thus seen to be equivalent to a "nodon" destruction operator.

By such a re-fermionization procedure, we can infer the properties of the vortex condensed phase, $\left\langle\Phi_{\sigma}\right\rangle \neq 0$, with $r_{\sigma}$ negative. This corresponds to taking $\mu$ negative and entering the molecular limits of the $p_{x}+i p_{y}$ superconductor. The critical point at $r_{\sigma}=0$, with massless but uncondensed $\Phi_{\sigma}$, is equivalent to the single massless Dirac field (with $\mu=0$ ) centered at zero momentum. Without recourse to re-fermionization, vortex condensation $\left\langle\Phi_{\sigma}\right\rangle \neq 0$ directly implies a mass for $a_{\mu}^{\sigma}$ and a vanishing spin Hall conductivity, $\sigma_{x y}^{s}=0$ - the correct value for the $p_{x}+i p_{y}$ molecular superconductor. This internal consistency gives us some confidence in the more general validity of the dual Ginzburg-Landau formulation.

An alternate route from the BCS to molecular limit is possible by implementing a duality transformation on the bosonic theory $\mathcal{L}_{\sigma}$, which interchanges the two phases. This can be achieved by expressing the bosonic threecurrent for the conserved spin $\left(\Phi_{\sigma}^{\dagger} \Phi_{\sigma}=\psi^{\dagger} \psi\right)$ as the curl of a gauge field, $\alpha_{\mu}$, and integrating out $a_{\mu}$. After shifting $\alpha_{\mu} \rightarrow \alpha_{\mu}+A_{\mu}^{\sigma}$ one thereby obtains,

$$
\mathcal{L}_{\text {Dual }}=\frac{1}{2}\left|\left(\partial_{\mu}-i \alpha_{\mu}-i A_{\mu}^{\sigma}\right) \phi\right|^{2}+r_{\phi}|\phi|^{2}+u|\phi|^{4}+\mathcal{L}_{c s}\left(\alpha_{\mu}\right) .
$$

Here $\phi$ creates a vortex in the field $\Phi_{\sigma}$. Notice that the dual theory has the same form as $\mathcal{L}_{\sigma}$ in Eq. 29, except for the absence of the Chern-Simons term in $A_{\mu}^{\sigma}$. Under duality, the "ordered" phase with $r_{\sigma}<0\left(\right.$ and $\left.\left\langle\Phi_{\sigma}\right\rangle \neq 0\right)$ maps into the "disordered" phase for $\phi$ with $r_{\phi}>0$. In this phase (the molecular limit) the dual theory correctly predicts $\sigma_{x y}^{s}=0$, due to the absence of the $A_{\mu}^{\sigma}$ ChernSimons term. In terms of the original Dirac field this duality is a particle/hole transformation, $\psi \rightarrow \psi^{\dagger}$, which changes the sign of the Dirac mass, $\mu \rightarrow-\mu$. The selfdual point where both $\phi$ and $\Phi_{\sigma}$ are critical, corresponds to the massless Dirac theory.

From the $p_{x}+i p_{y}$ molecular superconducting phase with $\sigma_{x y}^{s}=0$, it is possible to also proliferate and condense the charge vortex: $\left\langle\Phi_{\rho}\right\rangle \neq 0$, which describes a fully spin-gapped crystalline phase of spinless charge $e$ "holons".

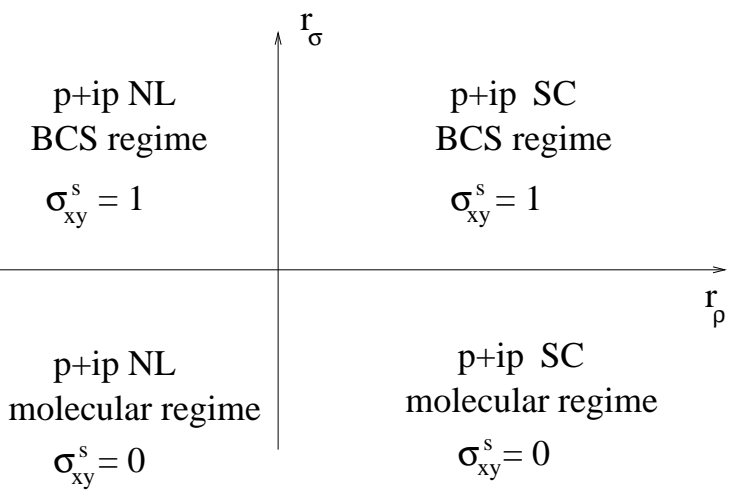

Fig. 1: The phase diagram in the $r_{\rho}-r_{\sigma}$ plane. Here, nodal liquid is denoted as $N L$, and $\sigma_{x y}^{s}$ is the spin Hall conductivity. 
In all four of the above phases (depicted schematically in the $r_{\rho}-r_{\sigma}$ plane in Fig. 1) time reversal invariance is spontaneously broken, reflecting the underlying $p_{x}+i p_{y}$ "pairing" symmetry. In each case, this symmetry breaking is taking place in the spin sector of the theory. The charge sector described by the simple Ginzburg-Landau theory (with no Chern-Simons term) is manifestly time reversal invariant. A natural question that arises is whether the spin-charge separated dual vortex theory in Eq. 17 can describe time reversal invariant phases, such as a $d_{x^{2}-y^{2}}$ superconductor or nodal liquid. Clearly a $d_{x^{2}-y^{2}}$ phase requires the breakdown of rotational invariance, either spontaneously or by the presence of an ionic potential. Moreover, since the $d_{x^{2}-y^{2}}$ superconductor exhibits gapless nodal excitations with finite momentum (on the Fermi surface in weak coupling BCS), it is necessary to access Fermi surface physics at non-zero momenta in the dual formulation. This can be readily achieved as we now discuss.

\section{V. "FERMI SURFACE", $P_{X}$, AND $D_{X^{2}-Y^{2}}$ PHASES}

\section{A. $p_{x}$ state}

In this section we imagine introducing an ionic potential with the lattice symmetry. The simplest case is a uniaxial perturbation, as might be appropriate, e.g. in a quasi-one-dimensional superconductor such as the cuprate ladder materials (the more interesting generalization to square symmetry will be returned to later). For simplicity, we take the symmetry axes along $x$ and $y$. Physically, it is clear that such a potential favors the formation of a real (non T-breaking) paired state such as $p_{x}$ or $p_{y}$. This can be seen by considering the twobody problem deep in the molecular limit. In the presence of the lattice potential, angular momentum is no longer a good quantum number (only discrete $\pm \pi$ rotations and reflections are symmetry operations) and the $p_{x} \pm i p_{y}$ states will generally be mixed. Coupling the two via a small "tunneling" perturbation, as appropriate for a weak ionic potential, splits the two initially degenerate levels into non-degenerate $p_{x}$ or $p_{y}$ eigenstates. The system then condenses into the lower of the two states. In the BCS limit the effects are more subtle, as we now illustrate.

As alluded to in the previous section, a distinguishing characteristic of the BCS theory of unconventional superconductors is the presence of gapless quasiparticle excitations at the intersections of the nodal lines of the pair wavefunction with the Fermi surface. For the $p_{x}$ case, this occurs at two antipodal points in momentum space $\mathbf{k}= \pm K \hat{\mathbf{y}}$, and the resulting gapless quasiparticles can be cast into the form of two Dirac species (see below). The multiplicity of Dirac fermions signals the emergence of two conserved U(1) charges in the low-energy limit. Essentially, because of phase space restrictions, the spin at each nodal point is separately conserved equivalently, one may view the two conserved charges as spin and (quasi-)momentum, the latter obtaining due to the absence of momentum-nonconserving umklapp processes for generically situated nodal wavevectors.

In the dual theory of Eq. 17, total spin conservation is manifest owing to the transverse nature of the spin current $J_{\mu}^{\sigma}=\frac{1}{2 \pi} \epsilon_{\mu \nu \lambda} \partial_{\nu} a_{\lambda}^{\sigma}$. Momentum conservation is also clear, but apparently on a very different footing it follows from the space-time Galilean invariance of the model. Apparently, to describe the BCS limit, a connection must be made between the internal (U(1) or $\mathrm{SU}(2))$ symmetry of the model and the external spacetime translational symmetry. It is quite remarkable that such a connection can indeed be clarified, as we now show.

To see how the finite momentum physics can emerge in the dual theory, first recall the quasiparticle structure of the $p_{x}+i \epsilon p_{y}$ superconductor in the BCS limit. This is described by Eq. 26 with $\mu>0$ and $\Delta_{\mathbf{k}}=v_{\Delta} k_{x}+i \epsilon k_{y}$ (only the symmetry, and not the particular form of this gap function is important in what follows). The low energy quasiparticles occur near $k_{x}=0, k_{y}= \pm K$, where $K \equiv \sqrt{2 m_{e} \mu}$. To focus on these two regions in momentum space, next define "slowly-varying" quasiparticle fields $f_{s \alpha}$ via

$$
\begin{aligned}
& c_{\uparrow}(\mathbf{x}) \sim \sum_{s= \pm} \frac{1}{\sqrt{2}}\left(f_{s \uparrow}-i f_{s \downarrow}\right) e^{i s K y}, \\
& c_{\downarrow}(\mathbf{x}) \sim \sum_{s= \pm} \frac{1}{\sqrt{2}}\left(i f_{-s \uparrow}^{\dagger}+f_{-s \downarrow}^{\dagger}\right) e^{i s K y} .
\end{aligned}
$$

One thereby obtains the Lagrange density

$$
\mathcal{L}_{p+i \epsilon p}=\sum_{s= \pm} f_{s}^{\dagger}\left[\partial_{\tau}+i v_{\Delta} \tau^{x} \partial_{x}+i s v_{F} \tau^{y} \partial_{y}+s m \tau^{z}\right] f_{s},
$$

where $v_{F}=K / m_{e}, m=\epsilon K$ and, following Eq. 27, we have introduced Pauli matrices $\vec{\tau}$ which act in the $\alpha=\uparrow, \downarrow$ space. Eq. 33 has the form of two decoupled Dirac equations, and thereby displays two manifest U(1) symmetries. Note from Eq. 32 that the $f_{\downarrow}$ fields are defined as hermitian conjugates relative to the $f_{\uparrow}$ fields in Eq. 31 . This implies that the overall $\mathrm{U}(1)$ rotation, $f_{s} \rightarrow e^{i \chi} f_{s}$, corresponds to spin $\left(S^{z}\right)$ conservation. The relative $\mathrm{U}(1)$, $f_{s} \rightarrow e^{i s \chi} f_{s}$, embodies instead translational symmetry, or momentum conservation.

The conserved densities are determined by Nöethers theorem:

$$
\begin{aligned}
& S_{+}^{z}=f_{+}^{\dagger} f_{+}=c_{K \uparrow}^{\dagger} c_{K \uparrow}-c_{-K \downarrow}^{\dagger} c_{-K \downarrow}, \\
& S_{-}^{z}=f_{-}^{\dagger} f_{-}=c_{-K \uparrow}^{\dagger} c_{-K \uparrow}-c_{K \downarrow}^{\dagger} c_{K \downarrow} .
\end{aligned}
$$

These are chiral spin densities, closely analogous to the chiral densities encountered in one-dimensional Fermi systems. The total spin density $S^{z}=S_{+}^{z}+S_{-}^{z}$. 
We are now in a position to bosonize the $p_{x}+i \epsilon p_{y}$ BCS model. The two flavors of Dirac particles $f_{ \pm}$may be traded for two complex boson fields $\Phi_{ \pm}$by attaching flux in a variety of ways. The most natural, however, is to introduce a single gauge field, attaching one flux quantum to each overall $\left(S^{z}\right) \mathrm{U}(1)$ charge. This choice ensures both that all the resulting bosonic fields commute at different space-time points and that the gauge field has the same physical meaning as the $a_{\mu}^{\sigma}$ defined previously. In particular,

$$
S^{z}=\frac{1}{2 \pi} \nabla \times \boldsymbol{a}^{\sigma}
$$

With this choice, the bosonized Lagrangian is

$$
\begin{aligned}
\mathcal{L}_{p+i \epsilon p}= & \sum_{s}\left|D_{0} \Phi_{s}\right|^{2}+v_{\Delta}^{2}\left|D_{1} \Phi_{s}\right|^{2}+v_{F}^{2}\left|D_{2} \Phi_{s}\right|^{2} \\
& +W\left(\Phi_{ \pm}\right)+\mathcal{L}_{c s}\left(a^{\sigma}\right)-\mathcal{L}_{c s}\left(A^{\sigma}\right)
\end{aligned}
$$

where $D_{\mu}=\partial_{\mu}-i\left(a_{\mu}^{\sigma}+A_{\mu}^{\sigma}\right)$. Here we have included an external spin gauge field $A_{\mu}^{\sigma}$ for bookkeeping purposes. The potential $W$ is dictated by symmetry to take the form

$$
\begin{aligned}
W\left(\Phi_{ \pm}\right)= & m^{2}\left[\left|\Phi_{+}\right|^{2}+\left|\Phi_{-}\right|^{2}\right] \\
& +u\left[\left|\Phi_{+}\right|^{2}+\left|\Phi_{-}\right|^{2}\right]^{2}+v\left|\Phi_{+} \Phi_{-}\right|^{2} .
\end{aligned}
$$

The final term in Eq. 37 is dictated by requiring $\sigma_{x y}^{s}=1$ in the $p_{x}+i \epsilon p_{y}$ phase where $m^{2}>0$.

Remarkably, the selection of two such non-zero "nodal" points occurs fairly naturally in our dual GinzburgLandau theory once uniaxial (rectangular) anisotropy is included. Consider the modified version of Eq. 29.

$$
\begin{aligned}
\mathcal{L}_{\sigma}= & \left|D_{0} \Phi_{\sigma}\right|^{2}+\left|D_{1} \Phi_{\sigma}\right|^{2}+c\left|D_{2} \Phi_{\sigma}\right|^{2} \\
& +\frac{d}{2}\left|\mathbf{D}^{2} \Phi_{\sigma}\right|^{2}+r_{\sigma}\left|\Phi_{\sigma}\right|^{2}+u_{\sigma}\left|\Phi_{\sigma}\right|^{4} \\
& +\mathcal{L}_{c s}\left(a^{\sigma}\right)-\mathcal{L}_{c s}\left(A^{\sigma}\right) .
\end{aligned}
$$

For simplicity, we have included only a single symmetrybreaking term, the coefficient $c<1$, which favors fluctuations along the $y$ axis over the $x$ axis. The coefficient $d>0$ is included for stability purposes.

As $c$ is decreased from one (zero anisotropy), the energy cost for fluctuations of $\Phi_{\sigma}$ with spatial variations along $y$ becomes more and more reduced. When $c$ changes sign and becomes negative, the lowest energy fluctuations bifurcate away from the origin in momentum space and move to two points $\mathbf{k}= \pm K \hat{\mathbf{y}}$, with $K=\sqrt{|c| / d}$. From this point on, it is appropriate to focus on the low-energy field configurations, viz

$$
\Phi_{\sigma}(\mathbf{x}) \sim \Phi_{+} e^{i K y}+\Phi_{-} e^{-i K y} .
$$

The physical meaning of Eq. 40 is clear from the above "reverse engineering" of the field content of the $p_{x}+i \epsilon p_{y}$ superconductor - compare with Eqs. 31-32. Naively inserting Eq. 40 in Eq. 39 and neglecting rapidly oscillating terms in the usual way gives an effective Lagrangian for the $\Phi_{\sigma \pm}$ fields. This has precisely the form of Eq. 37, with $v_{\Delta}^{2}=1+|c|, v_{F}^{2}=2|c|, m^{2}=r_{\sigma}-c^{2} / 2 d, u=u_{\sigma}$, and $v=2 u_{\sigma}$. These values (particularly $u$ and $v$ ) should, however, not be taken too seriously, as they certainly depend upon the simplistic treatment of fluctuations and higher-order terms.

With this identification in hand, we conclude that Eq. 39 provides a unified description of the $p_{x} \pm i p_{y}$ and $p_{x}$ states in an intrinsically anisotropic system. For $c<0$, the equivalence to Eq. 37 allows a refermionization to the form in Eq. 33. It is natural to associate the critical point of these equations $(m=0)$ with the spin structure of the $p_{x}$ superconducting state and its nodal-liquid/holon lattice counterpart. The refermionized double Dirac form in Eq. 33 is our primary result for the uniaxially anisotropic model.

Issues of time-reversal symmetry merit some discussion. Ideally, a general formulation of the problem should contain T-non-invariant terms only through spontaneous symmetry breaking. However, generically both Eq. 37 and Eq. 33 break T. In the fermionic formulation, Eq. 33, fortunately, the explicit symmetry breaking can be easily restored by requiring $m^{2}=0$. Furthermore, all Tpreserving perturbations of this form can be shown to be irrelevant, so that the $p_{x}$ state is locally stable. the point of view of Eq. 37, this is remarkable. Indeed, a direct mean-field analysis would suggest that a gapless state occurs only along the critical line $m^{2}=0$, requiring tuning of a parameter. These conclusions can be reconciled by noting that constraints upon Eq. 37 in a T-invariant system are not at all obvious. Only because of the ability to refermionize are we able to identify the critical line $m^{2}=0$ as containing a T-invariant manifold. If time-reversal is explicitly or spontaneously broken, a critical state is indeed non-generic, and the spectrum of Eq. 37 correctly reproduces that of the Dirac theory for small non-zero $m^{2}>0$ (i.e. $p_{x}+i \epsilon p_{y}$ with $\epsilon \ll 1$ ). Similar subtleties render the analysis of Eq. 37 problematic for $m^{2}<0$. We suspect that this region represents rather more exotic T-violating states, and do not consider it further.

Another physical route away from the BCS- $p_{x}$ phase is via a molecular $p_{x}$ state. Indeed, as we have argued above, deep into the molecular limit, uniaxial anisotropy guarantees a $p_{x}$ state. To tune through such a transition, we may imagine introducing a finite-range attraction into the 'non-crossing' model which favors tighter pair binding. In such a model the $p_{x}$-BCS state naturally undergoes a transition into a molecular $p_{x}$ state as the attraction is increased. From the conventional BCS point of view, we would expect such a transition to be described by taking the chemical potential through zero in a $p_{x}$ quasiparticle Hamiltonian, i.e. Eq. 26 with $\Delta_{k}=v k_{x}$. For this model, as $\mu$ passes from positive to negative, the quasiparticle nodes converge and coalesce at the origin, becoming massive for $\mu<0$. Precisely at the critical point, one expects a spectrum $\omega^{2} \approx v^{2} k_{x}^{2}+k_{y}^{4} / 4 m_{e}^{2}$. This 
unconventional non-Lorentz invariant form is precisely what is obtained at small wavevectors from Eq. 39 at the point where $c=r_{\sigma}=0$. Just as the gapless $p_{x}$ state occurs only along a line in mean-field theory but comprises a phase in the gauge model, it appears that the critical state at $c=r_{\sigma}=0$ (see Fig. 2) in reality forms a phase boundary despite appearing as a multicritical point in the mean-field treatment.

Given the existence of the $p_{x}$ molecular state, there must be another critical line separating this from the $p_{x} \pm i p_{y}$ molecular phase. As neither state contains gapless spin excitations or possesses a non-zero $\sigma_{x y}^{s}$, we have been unable to discriminate between them within the GL model. Viewed from the point of view of selfconsistent BCS theory, the transition would appear to be a simple first-order level crossing of two-particle bound states. Some preliminary modeling 8 suggests that a continuous transitions in the universality class of the quantum transverse-field Ising model is also possible, the Ising order parameter reflecting the presence or absence of $\mathrm{T}$ breaking. The full proposed phase-diagram in the spinsector is indicated in Fig. 2.

\begin{tabular}{c|c} 
p+ip SC & $\begin{array}{c}U_{\text {attractive }} \\
\text { molecular regime } \\
\sigma_{x y}^{s}=0\end{array}$ \\
$\begin{array}{c}\mathrm{p}_{x} \mathrm{SC} \\
\text { molecular regime } \\
\sigma_{x y}^{s}=0\end{array}$ \\
\hline $\mathrm{p}+$ ip SC & $\mathrm{p}_{x} \mathrm{SC}$ \\
BCS regime & $\mathrm{BCS}$ regime \\
$\sigma_{x y}^{s}=1$ & $\sigma_{x y}^{s}=0$
\end{tabular}

Fig. 2: The phase diagram as a function of increasing lattice potential with uniaxial anisotropy, $V_{\text {lattice, }}$ and short-range attractive pairing force, $U_{\text {attractive }}$.

\section{B. square symmetry and $d$-wave}

We have seen how a BCS-like $p_{x}$ state with gapless modes at non-zero momentum can emerge from the spin boson Lagrangian in Eq. 29 in the presence of uniaxial anisotropy. If the underlying crystal lattice has square symmetry, however, the $p_{x}$ or $p_{y}$ states are much less likely, and we expect instead that $d$-wave pairing is favored. Indeed, two particles in a fourfold rotationally invariant lattice potential interacting with a hard core will generally have a $d$-wave ground state in the limit of a strong potential. For a weak anisotropy, however, the $p_{x} \pm i p_{y}$ states are likely lower in energy. One thereby expects a transition upon increasing lattice coupling between p-wave and d-wave pairing. In the molecular limit this presumably occurs as a level crossing, i.e. a first or- der phase transition. In the BCS-like regime, however, the nature of the transitions or sequence of transitions between these states is less clear.

Rather than attempting to fully characterize this evolutionary process, we will content ourselves instead with determining the spin Lagrangian of the d-wave states themselves. Following the logic of the previous subsection, consider the simplest modification of Eq. 29 appropriate for square anisotropy,

$$
\begin{aligned}
\mathcal{L}_{\sigma}= & \left|D_{0} \Phi_{\sigma}\right|^{2}+c\left|D_{i} \Phi_{\sigma}\right|^{2}+d_{1}\left|\mathbf{D}^{2} \Phi_{\sigma}\right|^{2} \\
& +d_{2}\left(\left|D_{1}^{2} \Phi_{\sigma}\right|^{2}+\left|D_{2}^{2} \Phi_{\sigma}\right|^{2}\right)+r_{\sigma}\left|\Phi_{\sigma}\right|^{2}+u_{\sigma}\left|\Phi_{\sigma}\right|^{4} \\
& +\mathcal{L}_{c s}\left(a^{\sigma}\right)-\mathcal{L}_{c s}\left(A^{\sigma}\right)
\end{aligned}
$$

Here $d_{2}$ is a measure of the anisotropy, and low-energy excitations are pushed to finite momentum if $c$ becomes negative. For $c<0$ and $d_{2}<0$, the lowest energy modes occur at four points $\mathbf{k}=( \pm K, 0),(0, \pm K)$, precisely as expected for the low-energy excitations in a $d_{x y}$ superconductor (the momenta appropriate for $d_{x^{2}-y^{2}}$ are obtained for $\left.d_{2}>0\right)$ ! We fix $c<0$ and $d_{2}<0$ and consider decreasing $r_{\sigma}$.

Once again, it is appropriate to focus on the four slowly-varying fields $\Phi_{j s}(j=1,2, s= \pm)$, defined by

$$
\Phi_{\sigma}(\mathbf{x}) \sim \sum_{j s} \Phi_{j s} e^{i s \mathbf{K}_{j} \cdot \mathbf{x}}
$$

where $\mathbf{K}_{1}=(K, 0), \quad \mathbf{K}_{2}=(0, K)$, and $K=$ $\sqrt{|c| /\left(2\left(d_{1}+d_{2}\right)\right)}$. As for the $\mathrm{p}$-wave case, an effective theory can be developed for the $\Phi_{j s}$ fields. It takes the form

$$
\begin{aligned}
\mathcal{L}_{e f f}= & \sum_{j s}\left|D_{0} \Phi_{j s}\right|^{2}+v_{F}^{2}\left|D_{j} \Phi_{j s}\right|^{2}+v_{\Delta}^{2}\left|\epsilon_{j j^{\prime}} D_{j^{\prime}} \Phi_{j s}\right|^{2} \\
& +\tilde{W}\left(\Phi_{1,2 \pm}\right)+\mathcal{L}_{c s}\left(a^{\sigma}\right)-\mathcal{L}_{c s}\left(A^{\sigma}\right)
\end{aligned}
$$

Neither the precise values of $v_{F}, v_{\Delta}$ nor the form of $\tilde{W}$ is critical to this discussion. What is significant, however, is the fact that Eq. 43 takes the form of four interacting relativistic complex bosons coupled to a single U(1) Chern-Simons gauge field. As before, the effect of this gauge coupling is to attach identical flux to all spin quanta, transmuting these bosons into four species of Dirac fermions. Furthermore, the spin Hall conductivity $\sigma_{x y}^{s}=1$ in the massive phase (i.e. for $r_{\sigma}-c^{2} /\left(4\left(d_{1}+d_{2}\right)\right)>0$ in mean-field theory), as determined by the last term in Eq. 43. This is in agreement with the value in the $p_{x} \pm i p_{y}$ phase. Here, this value gives some indication of the structure of the signs of the four Dirac masses in the refermionized version of Eq. 43. Each massive Dirac equation gives a contribution of $\pm 1 / 2$ to $\sigma_{x y}^{s}$, depending upon the sign of its mass term, so apparently there must be three positive and one negative (or vice versa) masses in the Dirac theory. As the quadratic term in $\tilde{W}$ is tuned to zero, all four bose (and hence Dirac) fields are expected to go critical. This is 
the natural candidate for a time-reversal invariant point, and we speculate that it describes the nodal quasiparticles (nodons) in a $d_{\text {rg }}$ superconductor and relatives such as the nodal liquid.11 The appropriate fermionic representation for the nodons in these states was derived independently in Ref. 11. For $d_{x y}$ symmetry, it can be obtained as before from Eq. 26 by defining

$$
\begin{aligned}
& c_{\uparrow}(\mathbf{x})=\sum_{j s} d_{j s \uparrow} e^{i s \mathbf{K}_{j} \cdot \mathbf{x}}, \\
& c_{\downarrow}(\mathbf{x})=\sum_{j s} d_{j s \downarrow}^{\dagger} e^{-i s \mathbf{K}_{j} \cdot \mathbf{x}} .
\end{aligned}
$$

The (massless) nodon Lagrangian is then

$$
\begin{aligned}
\mathcal{L}_{\text {nodon }}= & \sum_{s= \pm} d_{1 s}^{\dagger}\left[\partial_{\tau}+s v_{F} \tau^{z} i \partial_{1}+s v_{\Delta} \tau^{x} i \partial_{2}\right] d_{1 s} \\
& +d_{2 s}^{\dagger}\left[\partial_{\tau}+s v_{F} \tau^{z} i \partial_{2}+s v_{\Delta} \tau^{x} \partial_{1}\right] d_{2 s},
\end{aligned}
$$

where as in earlier equations the $\vec{\tau}$ matrices act in the $\alpha=\uparrow, \downarrow$ subspace. For an alternate, explicitly $\mathrm{SU}(2)$ invariant formulation, see Ref. 11. Eq. 46 has the desired form of four Dirac equations (for $s= \pm, j=1,2$ ). The identification of Eq. 46 is supported by the excellent correspondence between the quantum numbers and momenta of the gapless modes of Eq. 43 and those of the nodons. In either case, there are four conserved U(1) currents, the charges (time components) of which are chiral spin densities, i.e. spin densities for particles with momenta along $\pm \mathbf{K}_{1}, \pm \mathbf{K}_{2}$. Mass terms taking Eq. 46 away from criticality can also be added, and take the form of $d_{i s}^{\dagger} \tau^{y} d_{i s}$ operators. The explicitly break time-reversal invariance, so that as for the $p_{x}$ phase, we expect the $d_{x y}$ (and analogously $d_{x^{2}-y^{2}}$ ) state to be locally stable.

Given the complexity of the arguments in this section, it seems appropriate to summarize what has been learned. We have studied how momentum space structure emerges from the bosonic Ginzburg-Landau theory of the spin sector. In doing so, we have not assumed (as in previous work on the nodal liquid) local superconductivity, but proceeded instead on very general grounds. Once the soft-modes of $\Phi_{\sigma}$ move to non-zero momenta, any incipient critical points can invariably be expressed in terms of multiple Dirac fields. When the associated Dirac masses vanish, a time-reversal invariant lagrangian is possible, and two such theories were identified with the $p_{x}$ and $d_{x y}$ nodal states. Furthermore, uniaxial and square lattice anisotropies were seen to favor appropriate critical states, even in very naive treatments of the Landau theory. These arguments provide a partial derivation of Eq. 46 for a generic time-reversal invariant d-wave (superconducting or nodal liquid) state, contingent only on the original hard-core assumption used to allow the Chern-Simons flux attachment.

\section{DISCUSSION}

In this paper, we have suggested that spin-charge separation is a generic consequence of strong repulsion between electrons in two-dimensions. We are driven inexorably to this conclusion by the following logic. (1) We note that if there are repulsive interactions which are strong enough to prevent electron trajectories from intersecting, then we may transmute the electrons into hardcore bosons interacting with a Chern-Simons gauge field which attaches flux to spin. (2) Since the up- and downspin boson currents are separately conserved, they can be written as the curls of two auxiliary gauge fields. (3) Using (1) and (2), we formulate an equivalent dual theory which is of Ginzburg-Landau form. The auxiliary gauge fields are minimally coupled to vortex fields. (4) The Ginzburg-Landau theory contains a $Z_{2}$ symmetry which, if broken by vortex condensation, leads to spin-charge confinement, translational symmetry-breaking, and 'conventional' ordered phases such as the AF and CDW. (5) In order to study phases with spin and charge physics at different scales and without translational symmetrybreaking, we contemplate $Z_{2}$-symmetric phases in which vortex pairs condense. A phase diagram of spin-charge separated states is the upshot of crossing the rubicon fed by these five tributaries.

By eschewing a conventional momentum-space approach which assumes a Fermi surface, we have constructed an effective field theory which does not fall under the rubric of Fermi liquid theory. The basic excitations of our theory are topological solitons in vortex condensates 24 . They are in no sense adiabatically connected to the electron and hole excitations of a free Fermi gas. They are also rather different from the 'holon' and 'spinon' conceptswhich are introduced to solve the Gutzwiller constraint25. These objects are stronglycoupled and do not appear to be soliton-like in character. The topological spinless charge $e$ excitation in our Ginzburg-Landau theory (a "holon") has an antiparticle with opposite charge, and at low energies can decouple from the neutral spin 1/2 Fermionic "nodon" excitation. Moreover, the "holon" already exists as a finite energy excitation within the Mott insulator - doping is not required.

Contrasting the present work with our earlier construction of the nodal liquid, we see that the "holon" field is identical to the charged soliton of the nodal liquid but the nodon - which descended from an assumed quasiparticle at the nodes of a $d_{x^{2}-y^{2}}$ superconductor - is now a concept which naturally arises from the spatially nonuniform softening of a vortex-anti-vortex pair field. Our nodon and holon fields are properly seen as analogous to the charge and spin solitons of the one-dimensional electron gas or the fractionally-charge quasiparticles in the quantum Hall effect.

Despite the surprising ease with which our dual Ginzburg-Landau formulation captures spin-charge sep- 
aration and superconductivity, the Fermi liquid phase seems to be missing. Generally, a dual vortex description of a Fermi liquid is possible, as illustrated nicely for the case of spinless electrons. After transforming to spinless bosons via Chern-Simons and implementing a duality transformation, one readily obtains a simple dual Ginzburg-Landau theory. This theory closely resembles Eq. 8, but with only a single vortex field, $\Phi$, which is minimally coupled to a single gauge field with ChernSimons dynamics. But more importantly, the theory is non-relativistic (i.e. there is a non-zero chemical potential) and the gauge field necessarily has a non-zero average, as the dual flux equals the number of electrons. So a vortex "vacuum" phase - the $p_{x}+i p_{y}$ superconductor for spinful electrons - is not accessible without spin. The Wigner crystal phase of spinless electrons corresponds simply to condensing the single vortex field, $\langle\Phi\rangle \neq 0$. In the Fermi liquid phase this vortex field must remain uncondensed, but with the vortices in a fluid state. This fluid of vortices presumably coexists with the fluid of particles (the Chern-Simons bosons which are the dual flux tubes) - the particle motion acting to scramble the vortex phase and vice versa.

By analogy, a description of a spinful Fermi liquid via the dual Ginzburg-Landau theory (Eq. 8) presumably requires an uncondensed but "critical" fluid of both up and down spin vortices. But this analogy is problematic for two reasons: Firstly, both vortex fields, $\Phi_{\alpha}$, are relativistic, with an equal number of positive and negative circulation vortices. But more importantly, in Eq. 8 the Chern-Simons term couples to the electron spin, effectively mediating a long-range statistical interaction between spin up and spin down electrons. In a spinful Fermi liquid, the up and down spin quasiparticle excitations should essentially propagate independently. It is for these reasons that we suspect the impossibility of describing a Fermi liquid phase in the dual formulation.

Why should the dual Ginzburg-Landau theory preclude a Fermi liquid, given that the only explicit assumption needed for its derivation was that opposite spin electron coordinates never coincide? In principle, the composite boson theory in Eq. 3 is exactly equivalent to a hard-core interacting fermion model, and therefore has a Fermi liquid phase in the presence of hard-core interactions only. The latter conclusion can be understood from the two-body problem of a small hard-core scatterer, from which it is seen that the s-wave scattering amplitude is small when the hard-core radius $a \ll k_{F}$. The subsequent manipulations leading to the dual formulation, however, do not treat this constraint carefully. Since the flux attachment procedure itself adds relative angular momentum to the wavefunction (c.f. Eq. 11), to accomodate a significant s-wave component requires incorporating $p$-wave effects in the composite bosons. Such non-local physics is easily missed, and presumably requires a much more subtle treatment of the duality transformation condition to capture it. When the repulsive interactions are sufficiently strong, however, we suspect the dual lagrangian to be adequate.

As we have argued, the presence of electrons spinning around one another is tantamount to significant (finite angular momentum) pairing correlations. The kinetic energy clearly favors lower angular momentum, which suggests the predominance of $p_{x}+i p_{y}$ pairing, at least in the absence of significant ionic potentials. Naively, this reasoning might suggest that quantum Hall systems with spinful electrons could exhibit high-temperature superconductivity. However, the presence of the strong orbital magnetic field presumably precludes this possibility. But there are quantum Hall systems which apparently dq exhibit high temperature "pseudo-spin" superfluidity 26 . In particular, double-layer quantum Hall systems with total filling $\nu=1$ do exhibit evidence of a "transverse superfluid" phase, with superfluid currents in the two layers flowing readily in opposite directions (effectively negating the effects of the magnetic field). It has been suggested that this phase will disorder via a finite temperature Kosterlitz-Thouless transition, with $T_{K T}$ in the $1 / 2$ Kelvin range - a superfluid transition driven by Coulomb repulsion.

The rather close analogy between this quantum Hall system and the $2 \mathrm{~d} p_{x}+i p_{y}$ superconductor (under the exchange of spin and charge) suggests a promising route for studying energetics. In the quantum Hall effect, variational wavefunctions have provided a powerful means of comparing various candidate ground states. Interchanging charge and spin, we therefore propose the following candidate wavefunction for the $p_{x}+i p_{y}$ superconductor:

$$
\Psi_{11 \overline{1}}=\prod_{i>j}\left(z_{i}-z_{j}\right)\left(w_{i}-w_{j}\right) \prod_{i, j}\left(\bar{z}_{i}-\bar{w}_{j}\right) e^{-\gamma \sum\left|z_{i}\right|^{2}+\left|w_{i}\right|^{2}} .
$$

Here, $z_{i}=x_{i}+i y_{i}$ denotes the complex coordinate of an up spin electron, and $w_{i}$ are down spin electron coordinates. The overbar denotes complex conjugation. As for quantum Hall wavefunctions the electron density is set by the exponential terms (with $\gamma$ proportional to the $2 \mathrm{~d}$ density). But this wave function involves both $z$ and it's complex conjugate $\bar{z}$. In this way, electrons moving with large angular momentum are avoided. In a slight abuse of quantum Hall notation, we refer to the wavefunction in Eq. 47 as a " $(1,1, \overline{1})$ " state. Readers familiar with Chern-Simons theory will recognize that Eq. 47 encapsulates the universal content ("K-matrix") of the effective field theory in Eq. 13, and is therefore a faithful representation of the $p_{x}+i p_{y}$ state. To make the "pairing" more explicit, it is instructive to use the Cauchy identity to re-express this wavefunction as

$$
\Psi_{11 \overline{1}}=\operatorname{Det}\left(\frac{1}{z_{i}-w_{j}}\right) \prod_{i, j}\left|z_{i}-w_{j}\right|^{2} e^{-\gamma \sum\left|z_{i}\right|^{2}+\left|w_{i}\right|^{2}} .
$$

Eq. 48 displays $\Psi_{11 \overline{1}}$ as the product of a BCS wavefunction (projected onto a state of definite electron number) 
with pair wavefunction $1 / z$ and a Jastrow factor. The Jastrow factor keeps up and down spin electrons apart appropriate for repulsively interacting $2 \mathrm{~d}$ electrons, but the first term evidently encapsulates pairing correlations adequate for superconductivity. It will be interesting to explore the energetics of $\Psi_{11 \overline{1}}$ in realistic models of repulsively interacting Fermions.

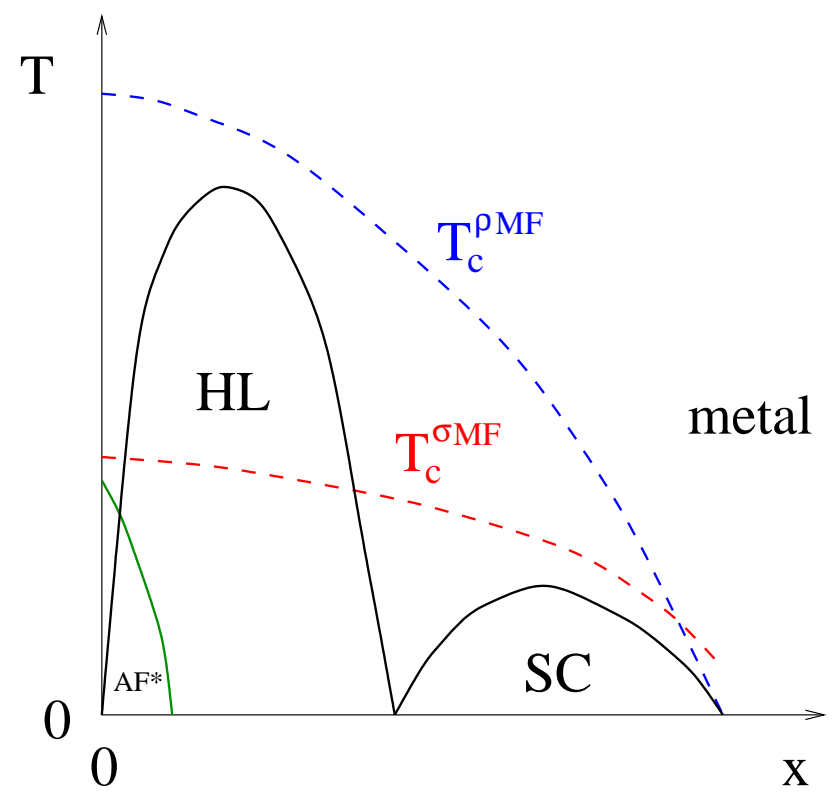

Fig. 3: Illustrative and schematic phase diagram of cuprate superconductors considered within the present dual Ginzburg-Landau framework. The (unconventional) Antiferromagnet, Holon Lattice, and SuperConducting phases are indicated by $\mathrm{AF}^{*}, \mathrm{HL}$, and SC, respectively.

In the canonical approach to strongly interacting electron systems near a Mott insulating phase, the very first step is to project onto a simpler tight binding model, oftentimes with one orbital per unit cell. When modeling the undoped cuprate superconductors a further projection to a reduced Hilbert space with one electron per site is usually adopted. The resulting spin Hamiltonian is much more tractable than the full system of interacting electrons, but we maintain that very important physics is irretrievably lost under these projections. For instance, the spin-charge separation that we access readily within our dual Ginzburg-Landau formulation of strongly interacting electrons in the $2 \mathrm{~d}$ continuum is certainly not present in the Heisenberg spin Hamiltonian. Put simply, it is exceedingly difficult to ascertain if spin and charge separate after projecting away the charge. We can, however, within our continuum approach, still describe Mott insulating physics by including a commensurate ionic potential which locks the charge order. In this way it is possible to describe Mott insulators which have gapped excitations and exhibit spin-charge separation. Indeed much, if not all, of the interesting physics accessible within the dual Ginzburg-Landau formulation is inaccessible, and probably not present, in the overworked $t-J$ model.

An apparent limitation of our approach is that we do not have a specific microscopic model which is described by our effective field theory and we do not know how strong the interactions must be to invalidate Fermi liquid theory. Nevertheless, we expect that, since many of the phases have excitation gaps, they should be stable to small perturbations and there should be a universality class of models which exhibit the same "universal" and robust properties (such as spin-charge separation). Indeed, as emphasized previously, the only formal requirement for obtaining the dual formulation is the "hardcore" part of the repulsion.

The physics of the Ginzburg-Landau formulation shares some tantalizing similarities with the cuprate high- $T_{c}$ materials. As discussed above, the crucial assumption of unbroken $Z_{2}$ symmetry appears most natural for systems with a large native disparity between charge and spin ordering scales. This is indeed the case for the cuprates near their half-filled Mott-insulating states. Precisely at half-filling, charge fluctuations begin to become quantized (acquire a gap) at very high temperatures of order $\mathrm{eV}$ (the Hubbard $U$ ), while for spins the characteristic ordering energy scale is significantly lower of order $J$ (with local moment formation ceuring somewhat higher). As in our previous work,11, 12 we hypothesize that this Mott phase comprises a spin-charge separated insulator described by Eq. 17. As the electron density at half-filling is commensurate with the underlying $\mathrm{CuO}_{2}$ plane periodicity, the charge sector (as well as the spin sector) is in effectively zero dual magnetic field.

The Ginzburg-Landau theories for charge and spin suggest a behavior in the temperature-doping plane illustrated in Fig. 3. At half-filling, both vortex fields have zero external flux and make transitions from their "normal" states at high temperatures to their "Meissner" states at low temperature. The associated meanfield transition temperatures, roughly $T_{c}^{\rho M F} \sim U$ and $T_{c}^{\sigma M F} \gtrsim J$, are shown in Fig. 3. Below $T_{c}^{\sigma M F}$, the spin boson $\Phi_{\sigma}$ begins to develop amplitude fluctuations, representing local moment formation. At somewhat lower temperatures this amplitude softens particularly near $( \pm \pi / 2, \pm \pi / 2)$, and the refermionized Dirac fields subsequently order into an $\mathrm{AF}^{*}$ phase (see Ref. 12 for details). Doping $x$ introduces a dual external flux $\bar{\nabla} \times \boldsymbol{a}^{\rho} \propto \phi_{0} x$ into the charge sector only - the spin boson $\Phi_{\sigma}$ is largely unaffected and in particular $T_{c}^{\sigma M F}$ presumably decreases only weakly. The dual flux in the Ginzburg-Landau theory for $\Phi_{\rho}$ introduces a dual mean-field " $H_{c 2}$ " line, or rapidly decreasing $T_{c}^{\rho M F}(x)$. Within a mean-field treatment a holon lattice phase would be expected below this line, in direct analogy with the Abrikosov flux lattice. But with fluctuations the holon lattice phase should be separated from $T_{c}^{\rho M F}(x)$ by a crossover regime analogous to the strongly-fluctuating "vortex liquid" state in type II superconductors. In the cuprate context, this is a regime of strong dynamical charge fluctuations and can 
be thought of as a "holon liquid", comprised of charge $e$ bosons. The $T_{c}^{\rho M F}(x)$ line then represents a crossover from a metallic phase above (with "unquantized" charge) to the holon liquid which manifests (dynamical) charge "quantization" in units of $e$ (cf. to dynamical "flux quantization" in the vortex liquid). Remarkably, our Ginzburg-Landau formulation suggests that spin-charge separation, effectively present below $T_{c}^{\rho M F}(x)$, can occur on very high interaction energy scales (eg. of order $U$ ). Upon further cooling the holon liquid one expects these bosons to condense, provided their density is sufficiently incommensurate with the underlying crystal potential to avoid charge ordering (into, for example, a holon lattice phase). This is the superconducting state, expected to be d-wave with a strong four-fold ionic potential. The predominant effect upon cooling through $T_{c}^{\sigma M F}$ above the superconducting phase, should be a reduction of low energy spin fluctuations and nodal formation in the electron spectral function, with a lesser effect in the charge sector due to weak spin-charge couplings. Although we emphasize that this is very much a preliminary application of the ideas of this paper, the picture in Fig. 3 is suggestive.

Our primary conclusion concerning the ubiquity of spin-charge separation and superconductivity driven by very strong repulsion has potential implications for a much broader class of other strongly interacting systems. Besides the cuprates, other systems include: the heavy fermion superconductors, quasi-one dimensional organic superconductors, low carrier 2DEG's with very lare $r_{s}$ in semiconductor MOSFET's and heterostructures 208 , superconductivify in $\mathrm{Sr}_{2} \mathrm{Ru} \mathrm{O}_{4}$ with possible $p_{x}+i p_{y}$ pairing symmetry 27, the normal and superfluid phases of 3 He (the A phase with a $p_{x}+i p_{y}$ pairing symmetry 29 ) and perhaps most intriguingly the magnetic states of solid 3-He 9.30. In many of these systems one is also very much interested in the full three dimensional limit, particularly for 3-He. Unfortunately, the Chern-Simons approach transforming fermions into bosons by flux attachment is restricted to strictly two-dimensional systems. But it is possible to transform between fermions and bosons in three dimensions by binding "statistical" magnetic monopoles to the particles 31 . Unfortunately, this introduces an unphysical internal statistical magnetic field (in contrast to the pure gauge coupling within Chern-Simons theory). But by attaching monopoles to spin, the monopole fields from the up spin electrons and the antimonopole fields from the down spin electrons will largely cancel (exactly on the average). Moreover, particle-vortex duality transformations are also possible in three-dimensions (ie. "electric-magnetic" duality), so it should be possible to obtain an entirely bosonic (but approximate) dual description of $3 \mathrm{~d}$ electrons with a 'non-crossing' constraint. Perhaps this approach might be useful in modeling some $3 \mathrm{~d}$ strongly correlated systems.

If, as we have suggested, strongly-interacting spin- $1 / 2$ fermions do not form a Fermi liquid, then our effec- tive field theory represents a new paradigm for correlated electron behavior. If, as we have further hypothesized, superconductivity is a prevalent attribute of the phases ensconced in our theory, then it is a paradigm which includes a new route to superconductivity. For these reasons, we submit that that our scenario could have far-reaching implications for the cuprate superconductors and other strongly-interacting electron systems.

We are grateful to Steve Girvin, T. Senthil and Doug Scalapino for illuminating discussions, and Mohit Randeria for gentle criticism of an overly hurried first draft. This research was generously supported by the NSF under Grants DMR-97-04005, DMR95-28578 and PHY9407194 .

${ }^{1}$ See, e.g. R. Prange and S.M. Girvin, eds., The Quantum Hall effect, Springer-Verlag, 1987; S. Das Sarma and A. Pinczuk, eds., Perspectives in quantum Hall effects : novel quantum liquids in low-dimensional semiconductor structures, Wiley, 1997.

${ }^{2}$ See M.P.A. Fisher and L.I. Glazman in Mesoscopic Electron Transport, page 331-373, edited by L.L. Sohn et. al. (Kluwer, 1997), and references therein.

3 J. G. Bednorz and K. A. Müller, Z. Phys. B 64, 189 (1986); For a recent review, see M. B. Maple, cond-mat/9802202 (unpublished).

${ }^{4}$ See, e.g., N. Grewe and F. Steglich, Handbook on the Physics and Chemistry of Rare Earths, eds. K.A. Gschneider and L. Eyring, 14, 343, Amsterdam, 1991; G.R. Stewart, Rev. Mod. Phys. 56, 755 (1984) and references therein.

${ }^{5}$ S. V. Kravchenko et al., Phys. Rev. B 51, 7038 (1995); D. Popović, A. B. Fowler, and S. Washburn, Phys. Rev. Lett. 79, 1543 (1997); Y. Hanien et al., Phys. Rev. Lett. 80, 1288 (1998); M. Y. Simmons et al., ibid, 80, 1292 (1998); S. J. Papadakis and M. Shayegan, Phys. Rev. B, June 15 (1998); J. Lam et al., Phys. Rev. B 56, R12741 (1997); P. T. Coleridge et al., ibid, 56, R12764 (1997).

${ }^{6}$ See, e.g. G. Baym and C. Pethick, in The Physics of Liquid and Solid Helium, Part 2, eds. J. B. Ketterson and K. Bennemann, Wiley, New York 1975; H. Godfrin and R.E. Rapp, Adv. Phys 44, 113 (1995); M. Siqueira, J. Nyeki, B. Cowan, and J. Saunders, Phys. Rev. Lett. 78, 2600 (1997).

${ }^{7}$ R. B. Laughlin, Phys. Rev. Lett. 50, 1395 (1983).

${ }^{8}$ L. Balents, M. P. A. Fisher, and C. Nayak, unpublished.

${ }^{9}$ See, e.g. F. Wilczek, Fractional Statistics and Anyon Superconductivity, World Scientific, 1990, and references therein.

${ }^{10}$ See, e.g. Eduardo Fradkin, Field Theories of Condensed Matter Systems, Addison-Wesley (1991), and references therein.

${ }^{11}$ L. Balents, M.P.A. Fisher, and C. Nayak, Int. J. Mod. Phys. B 12, 1033 (1998).

${ }^{12}$ L. Balents, M.P.A. Fisher, and C. Nayak, condmat/9811236.

${ }^{13} T$-violating superconducting ground states have been proposed for the cuprates in, for instance, R.B. Laughlin, Physica C 234, 290 (1994). 
${ }^{14}$ S. A. Kivelson and D. S. Rokhsar, Phys. Rev. B 41, 11693 (1990).

${ }^{15}$ See, eg. D. Vollhardt and P. Wolfe, The Superfluid Phases of Helium 3, Taylor and Francis (1990).

${ }^{16}$ P.W. Anderson, The Theory of Superconductivity in the High- $T_{c}$ Cuprates, Princeton, 1997.

${ }^{17}$ R. Shankar, Rev. Mod. Phys. 66, 129 (1994); J. Feldman, M. Salmhofer, E. Trubowitz, J. Stat. Phys. 84, 1209 (1996).

${ }^{18}$ C. Dasgupta and B.I. Halperin, Phys. Rev. Lett. 47, 1556 (1981); M.P.A. Fisher and D.H. Lee, Phys. Rev. B39, 2756 (1989); M. Peskin, Ann. Phys. 113, 122 (1978); P.O. Thomas and M. Stone, Nucl. Phys. B144, 513 (1978); X.G. Wen and A. Zee, Int. J. Mod. Phys. B 4, 437 (1990)

19 T. Senthil, J.B. Marston and M.P.A. Fisher, condmat/9902062.

${ }^{20}$ S.A. Kivelson, D.S. Rokhsar, and J.P. Sethna, Phys. Rev. B 35, 8865 (1987).

${ }^{21}$ For lack of a better term, we use 'holons' for our charge $e$ spinless solitons, even though our use of the term 'holons' is different from other uses of the term. For instance, our holons differ from the-holon introduced in the context of a doped $t-J$ model25, since ours come with both positive and negative charges and do not emerge by doping an antiferromagnetic insulator. Rather, our holons are the fundamental topological ("vortex") excitations in the $\Phi_{\rho}$ vortex condensate. In this sense, they similar to the holons of Kivelson, Rohksar, and Sethna 20 but our holons do not have a direct $1 \mathrm{D}$ analogue since they require a vortex condensate.

22 See, e.g. A.J. Leggett, in Proceedings of the XVIth Karpacz Winter School of Theoretical Physics, A. Pekalski, J. Przystawa, eds., Springer-Verlag, 1980; P. Nozieres and S. Schmitt-Rink, J. Low Temp. Phys., 59195 (1985).

${ }^{23}$ M. Randeria, J.-M. Duan, and L.-Y. Shieh, Phys. Rev. B 41, 327 (1990).

${ }^{24}$ For another perspective on 'topological doping', see S.A. Kivelson and V.J. Emery, Synthetic Metals, 80, (1996) 151.

${ }^{25}$ G. Baskaran, Z. Zou, P.W. Anderson, Solid State Comm. 63, 973 1987; Z. Zou, P.W. Anderson, Phys. Rev. B37, 627 (1988); N. Nagaosa, P.A. Lee, Phys. Rev. Lett. 64, 2450 (1990).

${ }^{26}$ See, e.g. the article by S.M. Girvin and A.H. MacDonald in the second reference in 1 .

${ }^{27}$ Y. Maeno et. al., Nature 372, 532 (1994); T.M. Rice and M. Sigrist, J. Phys. Cond.Matt. 7, L643 (1995); G. Baskaran, Physics B223, 490 (1996).

${ }^{28}$ A $p$-wave superconducting state in a 2DEG at large $r_{s}$ has been suggested in P. Philips, et al., Nature 395, 253 (1998). A spin liquid has been suggested in S. Chakravarty, et al., Phil. Mag., in press.

${ }^{29}$ D.M. Lee, Rev. Mod. Phys. 69, 645 (1997).

${ }^{30}$ M.C. Cross and D.S. Fisher, Rev. Mod. Phys. 57, 881 (1985).

31 A. Goldhaber, Phys. Rev. Lett. 36, 1122 (1982). 